\title{
Nanometer-scale Raman Spectroscopy of Neurons
}

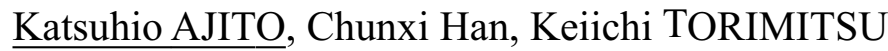

NTT Basic Research Laboratories, NTT Corporation, 3-1, Morinosato-Wakamiya, Atsugi, Kanagawa 243-0198, Japan.

Email: ajito@will.brl.ntt.co.jp http://www.brl.ntt.co.jp/people/ajito/index.html

Raman spectroscopy has medical applications in orthopedics, ophthalmology, and pathology, because it enable us to characterize fundamental biological molecules in cells, such as nucleic acid, peptide, proteins, and lipids. Recently many Raman spectroscopic techniques that use a near-field optical microscope, an evanescent wave illumination technique, or a multiphoton microscope have been developed to investigate single biological molecules or nanometer-sized cellular organelles. We developed a system that combines the laser trapping technique with Raman spectroscopy to investigate nanometer-sized cellular organelles using a near-infrared laser beam [1].

In this paper, we report the laser Raman trapping spectroscopy of nerve-ending particles called synaptosomes, which are found in the neurons in the brain. A schematic of a synapse and a sysnaptosome is shown in Fig. 1. The synaptosome is the simplest system, which comprises all the machinery for uptake, synthesis, storage, and exocytosis in neurons. The study of a single synaptosome is important because it will help us understand the roles of neurotransmitters at a single synapse level. Our system can capture a nanoparticle about $40 \mathrm{~nm}$ across by optical radiation pressure. This is much smaller than the size of a laser focal spot, about $1 \mu \mathrm{m}$ [2]. Therefore, we were able to trap synaptosomes, which are about $500-600 \mathrm{~nm}$.

The optical setup for our laser Raman trapping spectroscopy system is shown in Fig. 2 [3]. The near-infrared laser light from a titanium:sapphire laser focused on the sample with an objective lens with a numerical aperture of 1.3 for laser trapping is also used for the Raman spectroscopy, which makes optical arrangement very simple. The synaptosomes from the cerebral cortex of a 7- or 8-dayold Wister rat were prepared by homogenization and centrifugal separation using the Percoll gradient method.

Figure 3a shows a transmission micrograph of a synaptosome. The synaptosome is about $600 \mathrm{~nm}$ in diameter and contains many small synaptic vesicles of about $40 \mathrm{~nm}$, which contain neurotransmitters. The dark parts in the synaptosome indicate mitochondrias. The Raman spectrum of a single synaptosome laser-trapped in phosphate-buffered solution is shown in Fig. 3b. The circular pattern of interference laser light in the single synaptosome during laser-trapping is also shown in the spectrum. The background spectrum of the solution was subtracted from the spectrum. Two main peaks are observed in this spectral range. The peak at $1445 \mathrm{~cm}^{-1}$ is assigned to the $\mathrm{CH}_{2}$ deformation mode of lipids and the peak at $1657 \mathrm{~cm}^{-1}$ is assigned to the amide I mode of proteins. Also observed are two small peaks beside the peak of amide I. There are assigned to $\mathrm{COO}^{-}$stretching mode in the $\mathrm{NH}_{3}{ }^{+} \mathrm{COO}^{-}$group and $\mathrm{C}=\mathrm{O}$ stretching mode in the $\mathrm{COOH}$ group of glutamic acid. Glutamic acid is a typical neurotransmitter contained in synaptosomes. We confirmed that the peaks decreased with the firing of $\mathrm{Na}^{+}$-channels. The result indicates the laser Raman trapping spectroscopy can provide information about the function of neurons on a nanometer scale. The laser Raman trapping technique will probably be able to trap small synaptic vesicles and enable us to identify the many kinds of neurotransmitters in them without a staining process. This would be valuable information for understanding the function of neurotransmitters in the brain.

\section{References}

[1] K. Ajito, K. Torimitu, Lab Chip, 2 (2002) 11.

[2] K. Ajito, K. Torimitu Appl. Spectrosc. 56 (2002) 541.

[3] (a) K. Ajito, Appl. Spectrosc. 52 (1998) 339, (b) K. Ajito, M. Morita, K. Torimitsu, Anal. Chem. 72 (2000) 4721, (c)K. Ajito and K. Torimitsu, Trends Anal. Chem. 20 (2001) 255. 


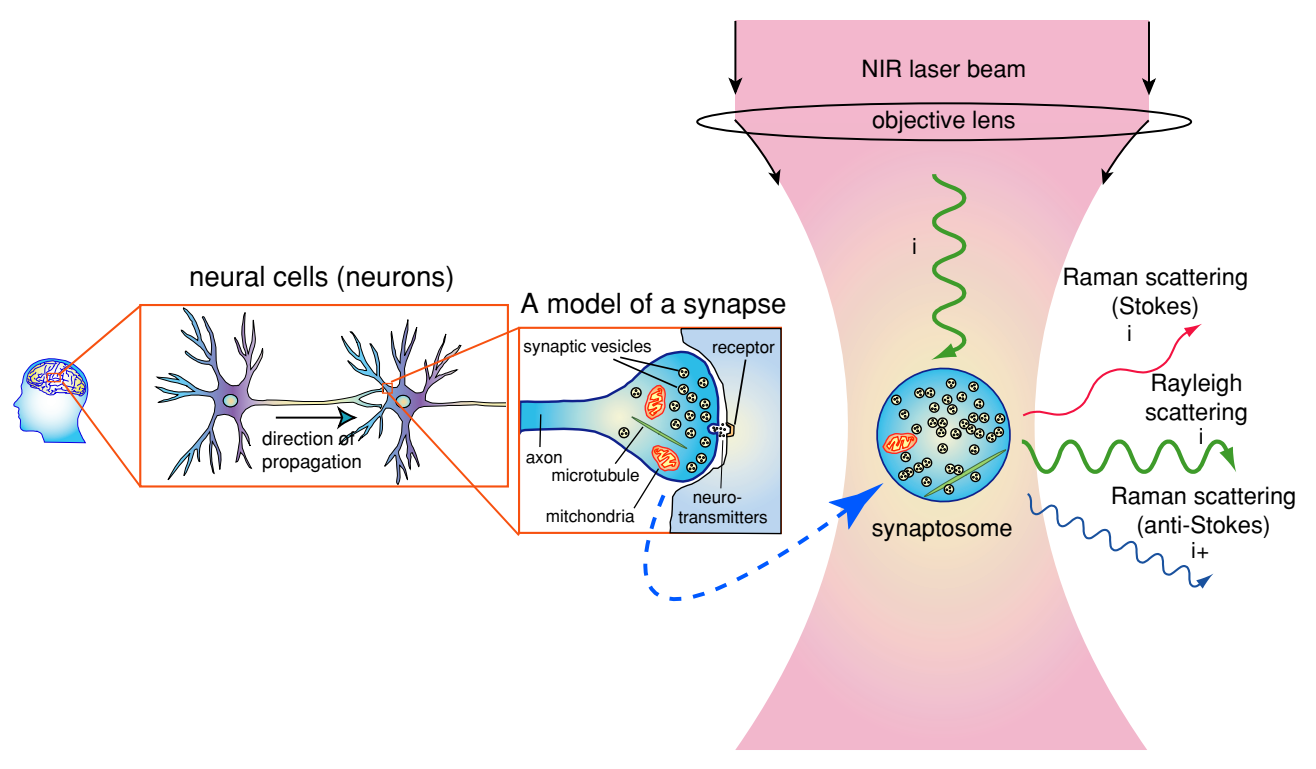

FIG. 1. Schematic of a synapse and the optical arrangement for laser trapping and Raman spectroscopy of a single synaptosome.

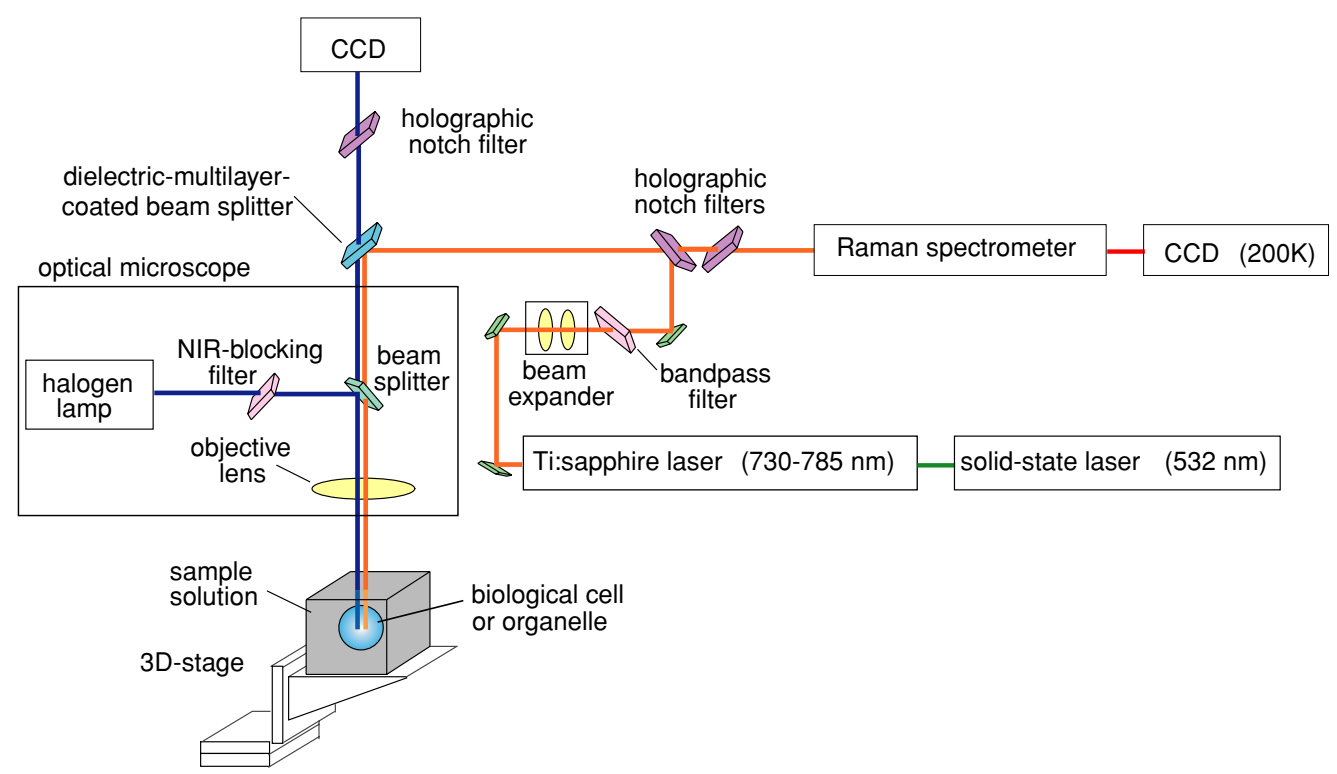

FIG. 2. Optical setup of the laser Raman trapping spectroscopy for biological samples.
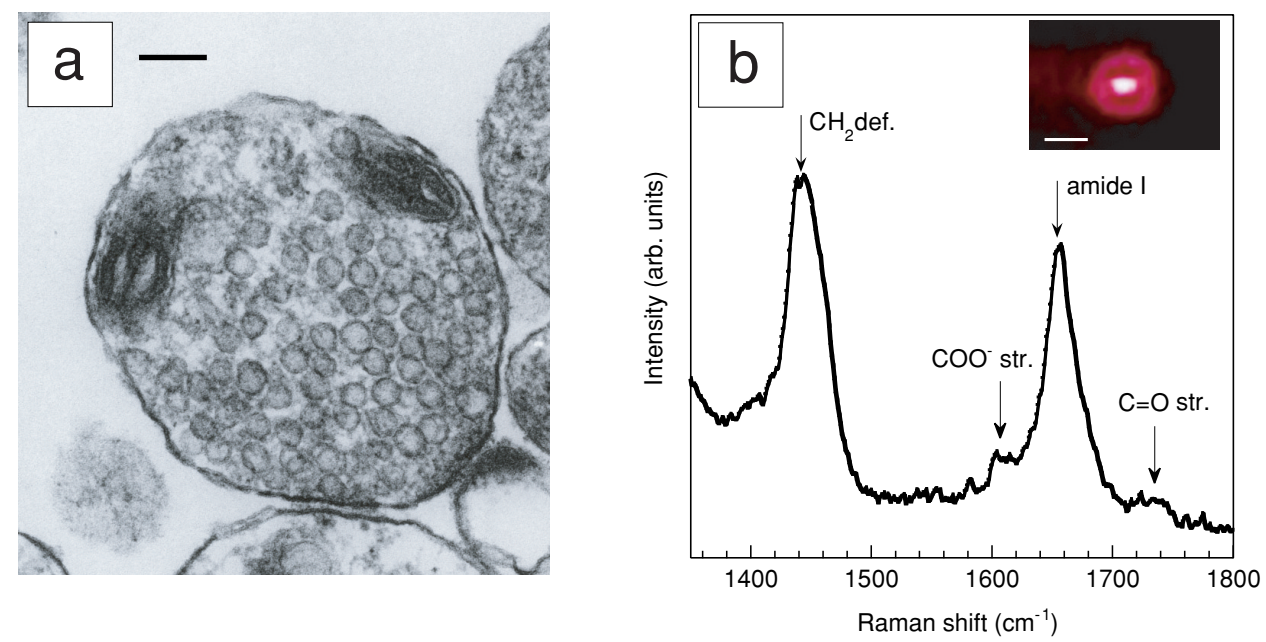

FIG. 3. (a) Transmission electron micrograph of a syanptosome (scale bar. $100 \mathrm{~nm}$ ). (b) A Raman spectrum of the laser-trapped single synaptosome in solution. The image in the spectrum is a interference laser light pattern reflected from the trapped synaptosome (scale bar. $1 \mu \mathrm{m}$ ). 